\title{
The Development of Evidence-Based Guideline for Diagnosis and Management of Headache in Korea
}

\author{
Sun Mi Kim¹, Young-Hoon Ko², Seoyoung Yoon ${ }^{3}$, Won Sub Kang ${ }^{4}$, Hye-Geum Kim ${ }^{5}$, Hye Youn Park \\ Cheolmin Shin ${ }^{7}$, Yoo Hyun Um ${ }^{8}$, Soyoung Youn ${ }^{9}$, Jae-Hon Lee ${ }^{10}$, Seung-Ho Jang ${ }^{11}$, Sang Won Jeon ${ }^{12}$, \\ Hong Jun Jeon ${ }^{13}$, Seockhoon Chung ${ }^{9}$, Jae-Won Choi ${ }^{14}$, Kyu-Man Han ${ }^{7}$, and Sang-Yeol Lee ${ }^{11 \bowtie}$ \\ 'Department of Psychiatry, College of Medicine, Chung-Ang University, Seoul, Republic of Korea \\ 2Department of Psychiatry, Korea University Ansan Hospital, Ansan, Republic of Korea \\ ${ }^{3}$ Department of Psychiatry, Catholic University of Daegu School of Medicine, Gyeongsan, Republic of Korea \\ ${ }^{4}$ Department of Psychiaty, College of Medicine, Kyung Hee University, Seoul, Republic of Korea \\ ${ }^{5}$ Department of Psychiatry, College of Medicine, Yeungnam University, Daegu, Republic of Korea \\ ${ }^{6}$ Department of Psychiatry, Seoul National University of Bundang Hospital, Seongnam, Republic of Korea \\ ${ }^{7}$ Department of Psychiatry, Korea University College of Medicine, Seoul, Republic of Korea \\ ${ }^{8}$ Deparment of Psychiatry, St. Vincent's Hospital, College of Medicine, The Catholic University of Korea, Suwon, Republic of Korea \\ ${ }^{9}$ Department of Psychiatry, Asan Medical Center, University of Ulsan College of Medicine, Seoul, Republic of Korea \\ ${ }^{10}$ National Rehabilitation Center, Seoul, Republic of Korea \\ ${ }^{11}$ Department of Psychiatry, Wonkwang University School of Medicine and Hospital, Iksan, Republic of Korea \\ ${ }^{12}$ Department of Psychiatry, Kangbuk Samsung Hospital, Sungkyunkwan University School of Medicine, Seoul, Republic of Korea \\ ${ }^{13}$ Department of Psychiatry, Konkuk University Medical Center, Seoul, Republic of Korea \\ ${ }^{14}$ Department of Neuropsychiatry, Eulji University School of Medicine, Seongnam, Republic of Korea
}

Objective We aimed to develop the clinical guideline for headache by the systematic review and synthesis of existing evidence-based guidelines. The purpose of developing the guideline was to improve the appropriateness of diagnosis and treatment of headache disorder, and consequently, to improve patients' pain control and quality of life. The guideline broadly covers the differential diagnosis and treatment of tension-type headache, migraine, cluster headache, and medication-overuse headache.

Methods This is a methodological study based on the ADAPTE methodology, including a systematic review of the literature, quality assessment of the guidelines using the Appraisal of Clinical Guidelines for REsearch \& Evaluation II (AGREE II) Instrument, as well as an external review using a Delphi technique. The inclusion criteria for systematic search were as follows: topic-relevant, up-to-date guidelines including evidence from within 5 years, evidence-based guidelines, guidelines written in English or Korean, and guidelines issued by academic institutions or government agencies.

Results We selected five guidelines and conducted their quality assessment using the AGREE II Instrument. As a result, one guideline was found to be eligible for adaptation. For 13 key questions, a total of 39 recommendations were proposed with the grading system and revised using the nominal group technique.

Conclusion Recommendations should be applied to actual clinical sites to achieve the ultimate goal of this guideline; therefore, followup activities, such as monitoring of guideline usage and assessment of applicability of the recommendations, should be performed in the future. Further assessment of the effectiveness of the guideline in Korea is needed.

Psychiatry Investig 2019;16(3):199-205

Key Words Headache, Guideline, Tension-type headache, Migraine, Cluster headache, Medication-overuse headache.

\section{INTRODUCTION}

Headache is a very common symptom experienced by $70^{-}$ $80 \%$ of the population. ${ }^{1}$ According to the Healthcare Bigdata
Hub statistics of the Health Insurance Review and Assessment Service, the number of patients who visited a doctor for headache increased from 671,156 to 867,569 in the past 7 years (2010-2016), an increase of $29 \%{ }^{2}$

Received: August 30, 2018 Revised: November 1, 2018 Accepted: November 23, 2018

$\square$ Correspondence: Sang-Yeol Lee, MD, PhD

Department of Psychiatry, Wonkwang University School of Medicine and Hospital, 895 Muwang-ro, Iksan 54538, Republic of Korea

Tel: +82-63-859-1052, Fax: +82-63-857-1043, E-mail: psysangyeol@hanmail.net

(c) This is an Open Access article distributed under the terms of the Creative Commons Attribution Non-Commercial License (https://creativecommons.org/licenses/by-nc/4.0) which permits unrestricted non-commercial use, distribution, and reproduction in any medium, provided the original work is properly cited. 
Headache disorders are classified as primary and secondary headaches. ${ }^{3}$ To date, the etiology of primary headache disorder has not been clearly elucidated, and such headache disorders are classified according to clinical features. Tension-type headache, migraine, and cluster headache are the most common primary headache disorders. ${ }^{4}$ Secondary headache disorder is caused by other underlying diseases, such as headaches due to head and neck trauma and disease, vascular disease involving the head or neck, and substance abuse or withdrawal. ${ }^{5}$ Among secondary headache disorders, medication-overuse headache is the most common in people receiving medications due to primary headache disorders. ${ }^{6}$ The medical and social burden of headache is mainly caused by primary headache disorders and medication-overuse headache. ${ }^{1}$

Despite the high prevalence and social costs of headache disorders, there is no domestic clinical guideline that covers evaluation, differential diagnosis, and treatment of major headache disorders such as tension-type headache, migraine, cluster headache, and medication-overuse headache. Therefore, there is a large variation in the treatment of patients with the same type of headache, such as the type of investigation, medication, and non-pharmacological management according to the medical institution, the specialty of a doctor, and individual preference of doctors. At this point, the development of a headache treatment guideline through systematic review and synthesis of existing evidence is very important to maintain the professionalism, systematization, and continuity of headache care, as well as to avoid unnecessary cost of medical care.

In this paper, we describe the process of developing the clinical guideline for headache by the systematic review and synthesis of existing evidence-based guidelines.

\section{METHODS}

\section{Organizing Committee}

An Organizing Committee was formed at first to plan and oversee the guideline development process. The Organizing Committee consisted of 18 persons recommended by the Korean Psychosomatic Society. The Organizing Committee oversees the entire adaptation development process. In other words, they set the scope of development of the guideline, develop the methodology, form a Working Committee, review the development process, disseminate the guidelines, and formulate implementation strategies. The study protocol was approved by the Institutional Review Board of the Chung-Ang University Hospital (approval number: 1710-006-16113).

\section{The ADAPTE process}

Since there are already high quality evidence-based guidelines for headaches overseas, the Organizing Committee de- cided to use the ADAPTE approach endorsed by the Guidelines International Network. ${ }^{7}$ Recognizing that considerable resources are required for guideline development, the ADAPTE process was followed to leverage existing guidelines and diminish duplication of effort. The ADAPTE process uses a systematic approach to apply guidelines generated from a single setting in different cultural and organizational settings. The ADAPTE process has been used in many other organizations to develop guidelines worldwide. It has three phases: set-up, adaptation, and finalization (Figure 1).

\section{Set-up phase}

During the set-up phase, the Organizing Committee set the scope of development of the guideline, formed a Working Committee, and planned further steps. The Working Committee consisted of 16 persons who were recommended by the Organizing Committee as well as three research methodology experts. The Working Committee conducts substantive guideline adaptation processes, such as selecting health questions and keywords, screening the searched guidelines, drafting the recommendations and evidence of the health questions, drafting the recommendations, and finalizing the recommendations. Conflict of interests were declared by each member of the Organizing and Working Committees, and updated at each meeting.

\section{Adaptation phase}

Define health questions

During the adaptation phase, there was a discussion to de-

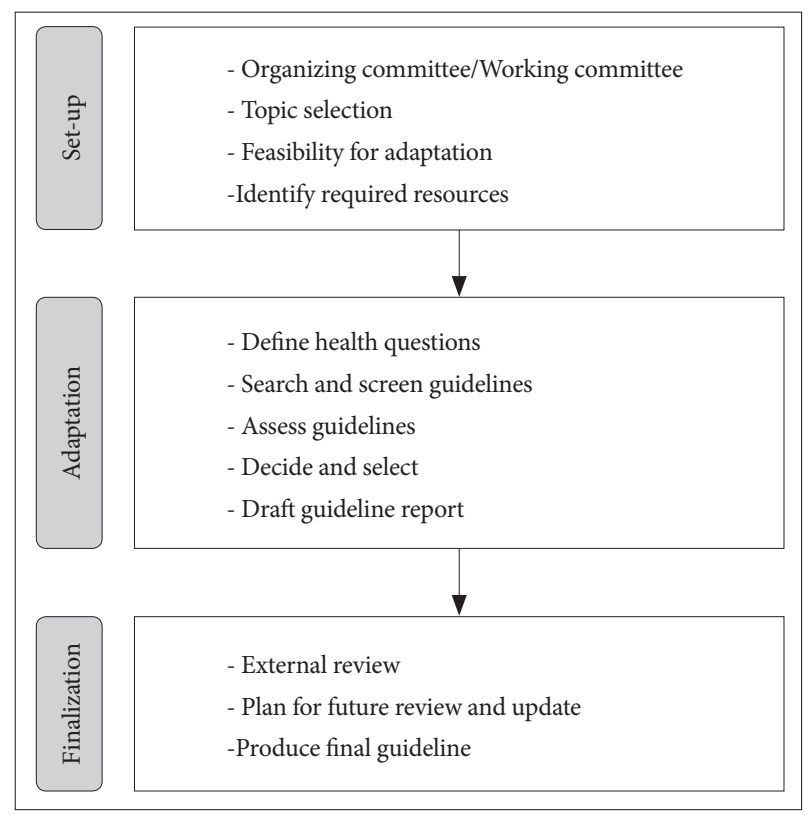

Figure 1. Three phases of the ADAPTE process. 
fine the Population, Intervention, Professionals, Outcomes and Health settings (PIPOH) scheme more clearly (Table 1). The Working Committee agreed that the guideline broadly covers the differential diagnosis and treatment of tension-type headache, migraine, cluster headache, and medication-overuse headache. Both pharmacological management and psychosocial intervention of headache were considered important topics for inclusion. Target users of this guideline are general practitioners and related specialists (psychiatrists, neurologists, neurosurgeons, family medicine doctors, etc.).

\section{Search and screen guidelines}

The existing guidelines were searched for the present adaptation. We searched various domestic and international databases for guidelines in clearinghouses, such as the National Guideline Clearinghouse and Guidelines International Network, and in the websites of guideline developers, such as the National Institute for Health and Care Excellence (NICE) and Scottish Intercollegiate Guidelines Network. We also searched for guidelines in PubMed, Embase, Cochrane, Agency for Healthcare Research and Quality, and National Comprehensive Cancer Network. We searched for guidelines which dealt with at least one headache disorder among tension-type headache, migraine, cluster headache, and medication-overuse headache. The inclusion criteria were that the guidelines needed to be evidence-based; published after November 2012, including the evidence of recent 5 years at the time of development or renewal; written in English or Korean, and developed by an academic institution or government agency. In addition, for the sake of uniformity, only guidelines that specified accurate diagnostic criteria were selected, and the most recent version was selected when there were renewal versions. The exclusion criteria were that the guidelines did not include information on the quality of evidence or strength of recommendation, were published without references, and were meant for the care of children and adolescents only.

\section{Assess guidelines}

The guidelines identified after searching and screening were evaluated in various aspects, such as the quality, up-to-date status, content, and acceptability/applicability. The quality of these guidelines was reviewed and evaluated using Korean version of the Appraisal of Guidelines for Research and Evaluation II (K-AGREE II) tool which consists of 23 items in 6 assessment domains, and each item is assigned a score on the 7-point Likert scale. ${ }^{8}$ To improve the reliability of the K-AGREE II tool, four members of the Working Committee evaluated the guidelines and then coordinated their views on the results of each evaluation. Based on the results of the K-AGREE II evaluation, the standardization score for each evaluation domain was calculated, and the guidelines which scored more than $50 \%$ in the "Rigour of development" domain were selected as the latest guidelines for content evaluation. In order to investigate the up-to-date status of the guidelines for adaptation, the date of publication of the guidelines and the date on which the latest evidence was searched were examined. In addition, the contents of the existing guidelines were evaluated if they covered the contents of the new guideline being developed. Acceptability/applicability tests were performed to assess the extent to which the guidelines and recommendations could be used in practice in the Korean setting. Acceptability refers to whether the guideline can be accepted in the Korean context, and applicability refers to whether the guideline can be applied to clinical practice in the Korean context.

\section{Decide and select}

Recommendations in accordance with the 13 health questions were extracted from the selected guidelines as the target

Table 1. Population, Intervention, Professionals, Outcomes and Health settings (PIPOH)

\begin{tabular}{|c|c|c|}
\hline PIPOH & Category & Contents \\
\hline Population & Headache in adults above 19 years of age & $\begin{array}{l}\text { Tension-type headache } \\
\text { Migraine } \\
\text { Cluster headache } \\
\text { Medication-overuse headache }\end{array}$ \\
\hline Intervention & $\begin{array}{l}\text { Pharmacological treatment } \\
\text { Psychosocial treatment }\end{array}$ & \\
\hline Professions & General practitioners and related specialist & (psychiatrists, neurologists, neurosurgeons, family medicine doctors, etc.). \\
\hline Outcome & $\begin{array}{l}\text { Patient outcome } \\
\text { System outcome }\end{array}$ & $\begin{array}{l}\text { Pain control and improvement of quality of life } \\
\text { Improvement of the appropriateness of diagnosis and treatment of headache } \\
\text { disorder }\end{array}$ \\
\hline Healthcare setting & Medical institutions & $\begin{array}{l}\text { Primary medical institutions, outpatient treatment institutions, and inpatient } \\
\text { treatment institutions }\end{array}$ \\
\hline
\end{tabular}


of adaptation. The Working Committee members carefully examined the evidence, acceptability and applicability, and acceptability/applicability in the Korean context of each recommendation, in accordance with the ADAPTE process.

The Working Committee drafted the recommendations by extracting recommendations on health questions from the selected guidelines as the target of adaptation. After reviewing the evidence for the draft of the recommendations, the Working Committee conducted preliminary discussions on the acceptability/applicability as well as the clarity of the recommendations. At this meeting, modifications were proposed through informal consensus among the Working Committee members, based on the recommendation that the original form was difficult to be accepted as the recommendations were meant for adaptation, and hence, revision was necessary.

After reviewing the draft of the recommendations, the Working Committee decided which recommendations to accept, which to discard, and which were suitable but needed to be modified. When modifying existing recommendations, the Working Committee was careful not to modify the recommendation to such an extent that it was no longer in accordance with the evidence on which it was based. Modifications were proposed through informal consensus, and then formal consensus was reached using the Nominal Group Technique (NGT).

The final list of recommendations was presented to the Organizing Committee as well as the Working Committee at an in-person consensus meeting. For each recommendation, formal consensus was reached using the NGT, and it was accepted when a consensus level of $70 \%$ or more was achieved. The NGT was performed according to the method described in the Handbook for Clinical Practice Guideline Developer. ${ }^{9}$ Recommendations required agreement by $70 \%$ of the group to be included in the Korean guideline for headache.

\section{Draft guideline report}

Based on the accepted recommendations, the Working Committee drafted the guideline by allocating them to subcategories. The draft guideline consisted of three chapters: 1) Introduction, including the background, necessity, scope, and purpose of the guideline; 2) the method, including the composition of the committee, selection of health questions, search for the guidelines, and evaluation of the guidelines; and 3) the recommendations. Annexures included the Korean-English medical terminology and abbreviations used in the guidelines, the form of interest declaration, the results of interest declaration, existing evidence search process, the results of K-AGREE II implementation, the results of up-to-date status test, and the acceptability/applicability test form and results. The Organizing and Working Committees convened to review the format and content of the draft in detail and approve the draft.

\section{Finalization phase}

During the finalization phase, an external review by experts, further planning for future review and update, and the production of a final guideline were conducted.

\section{External review}

The members of the Review Committee composed of experts in related fields. Headache is diagnosed and treated by general physicians and professionals from various specialties (psychiatrists, neurologists, neurosurgeons, family medicine doctors, etc.) in primary medical institutions, outpatient treatment institutions, and inpatient treatment institutions. Therefore, the Review Committee (consensus group) was composed of ten specialists, three psychiatrists, one neurologist, three neurosurgeons, and three family medicine doctors. The Review Committee reviewed the final draft recommendations and participated in the expert panel survey using Delphi method. The Delphi method was followed in accordance with the method described in the Handbook for Clinical Practice Guideline Developer. ${ }^{9}$ Each recommendation was finally confirmed if a consensus level of $70 \%$ or more was reached in the Delphi method. The final guidelines were drawn up after final draft recommendations were confirmed by the Delphi method.

\section{Plan for future review and update}

In the future, a revision will be performed when it is judged that new knowledge regarding the diagnosis, evaluation, and treatment of headache have been accumulated, and revision is necessary.

\section{RESULTS}

\section{Set-up phase}

The Organizing Committee consisted of 18 persons and the Working committee consisted of 16 persons. All members declared they have no conflict of interests.

\section{Adaptation phase}

\section{Define health questions}

To reproduce the Population, Intervention, Professionals, Outcomes and Health setting, 13 health questions regarding the evaluation, diagnosis, and management of headache were derived. The categories of health questions have been presented in Table 2.

\section{Search and screen guidelines}

The screening procedure for the guidelines and results are 
Table 2. Categories of health questions and related recommendations

1. Evaluation of headache
1.1 Additional evaluation, inspection, or referral criteria
1.2 Use of headache diary for assessment and diagnosis
2. Diagnosis of headache
2.1 Diagnosis of primary headache disorders and medication-
overuse headache
2.2 Brain imaging in diagnosis and treatment of primary
headache disorders
3. Treatment of headache
3.1 Use of headache diary for treatment
3.2 Acute treatment of tension-type headache
3.3 Preventive treatment of tension-type headache
3.4 Acute treatment of migraine with or without aura
3.5 Preventive treatment of migraine with or without aura
3.6 Acute treatment of cluster headache
3.7 Prophylactic treatment of cluster headache
3.8 Treatment of medication-overuse headache
3.9 Psychosocial treatment of primary headache disorders

provided as the Preferred Reporting Items for Systematic Reviews and Meta-Analyses (PRISMA) flow diagram in Figure 2.

\section{Assess guidelines}

Five existing guidelines were identified to be potentially suitable for adaptation. Based on the results of the K-AGREE II evaluation, three of five existing guidelines scored more than $50 \%$ in the "Rigour of development" domain. Among the three existing guidelines, only the NICE National Clinical Guideline Number 150 (CG150) included the evidence of recent 5 years at the time of development or renewal, according to the up-to-date status test. Therefore, finally, the 2015 updated version of the "Headaches in over 12s: diagnosis and management" developed by NICE (NICE CG150) was selected as the target of adaptation. ${ }^{10}$ We have made a license agreement with NICE for adaptation of content.

\section{Decide and select}

Recommendations in accordance with the 13 health questions were extracted from the NICE CG150. The Working committee drafted recommendations by extracting recommendations on health questions from the NICE CG150. Common reasons for modification were differences in resources between United Kingdom and Korea.

Although many recommendations were accepted without modification, several were modified according to the Korean context. The recommendation of the NICE CG150, "Discuss the need for neuroimaging for people with a first bout of clus- ter headache with a GP with a special interest in headache or a neurologist," was modified to "Discuss the need for neuroimaging for people with a first bout of cluster headache with a specialist in the relevant department." Korea has a higher proportion of specialists (73\%) compared with the Organisation for Economic Co-operation and Development (OECD) average $(63 \%) .{ }^{11}$ Due to the high accessibility of specialists, it was deemed that the phrase "a specialist in the relevant department" was more appropriate. The decision for the modification was made through informal consensus between the Organizing and Working Committees, and confirmed with NGT, which is a formal consensus method. In the NICE CG150, anti-emetic agents and non-oral preparation of metoclopramide or prochlorperazine are recommended for the management and acute treatment for migraine. However, this is difficult to apply in domestic practice because migraine treatment is not included in the permission for use of anti-emetics issued by the Ministry of Food and Drug Safety, and is not covered by insurance. Therefore, the Organizing and Working Committees agreed that there is no great limitation on the domestic acceptability of this recommendation, but it is difficult to apply it. The use of amitriptyline for migraine prophylaxis and verapamil for cluster headache prophylaxis were also difficult to apply in domestic practice because these medications are not included in the permission issued by the Ministry of Food and Drug Safety, and are not covered by insurance. The recommendation of the NICE CG150 to "Offer oxygen and/or a subcutaneous or nasal triptan for the acute treatment of cluster headache" was also discussed. Currently, it is possible to supply oxygen in some emergency rooms in Korea, but home or ambulatory oxygen generator is not available yet. In addition, subcutaneous or nasal triptan is currently not available in Korea. Accordingly, the Organizing and Working Committees agreed that there was no limitation on the acceptability of this recommendation, but it was difficult to apply it. Therefore, this recommendation will be effective only when the appropriate treatment method becomes available in the future. All these considerations have been described in the guideline, because it was agreed that the guideline should provide up-to-date knowledge and consensus to the target population regardless of its applicability, and the application of the recommendation may be feasible if the resource becomes available later.

Finally, a total of 39 recommendations regarding the 13 health questions were presented to the Organizing and Working Committees, and evaluated in the in-person consensus meeting. All recommendations and modified recommendations reached a consensus level of over $70 \%$ in the first round of NGT.

\section{Finalization phase}

The Delphi method was conducted through email from June 


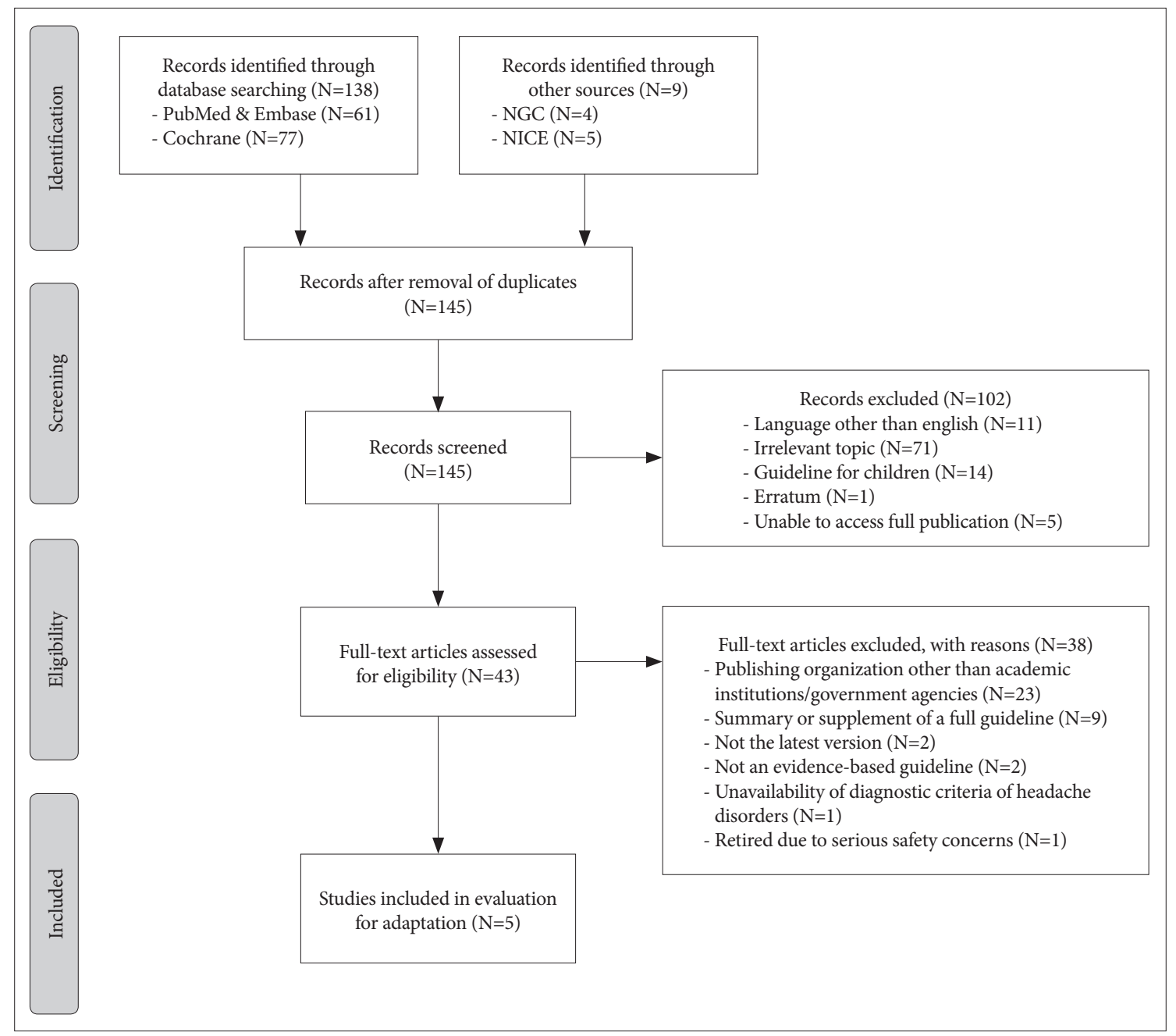

Figure 2. The Preferred Reporting Items for Systematic Reviews and Meta-Analyses (PRISMA) flow diagram.

11, 2018 to July 21, 2018, and each recommendation was finally confirmed if a consensus level of $70 \%$ or more was reached. All recommendations reached agreement levels of over $70 \%$ in the second round of the Delphi method. Guideline adaptation resulted in 39 final recommendations.

The guidelines were officially launched at the Annual Scientific Meeting of the Korean NeuroPsychiatric Association, held at Busan in October 2018.

\section{DISCUSSION}

Guidelines for assessing and managing headache in adults have been developed for the Korean setting. The purpose of developing the "Evidence-based clinical guideline for diagnosis and management of headache in Korea" was to improve the appropriateness of diagnosis and treatment of headache disorder, and consequently, to improve patients' pain control and quality of life. The guideline broadly covers the differential di- agnosis and treatment of tension-type headache, migraine, cluster headache, and medication-overuse headache. Target users of this guideline are general practitioners and related specialists (psychiatrists, neurologists, neurosurgeons, family medicine doctors, etc.).

Guideline development is a costly and time-consuming challenge. ${ }^{12}$ Therefore, we chose the ADAPTE methodology for the adaption of evidence-based international guideline recommendations. The main benefit of using the ADAPTE process is financial. De novo guideline development requires considerable resources to perform evidence reviews, and may take years to complete. The ADAPTE process leverages existing guidelines and reduces duplication of effort. The weakness of the ADAPTE process is the issue regarding up-to-date status. Adaptation of guideline also takes time to complete, although it is less time-consuming de novo guideline development; therefore, the evidences based on the original recommendations may be outdated by the time the adapted guidelines are pub- 
lished.

We came across a large number of guidelines in the first phase of our search strategy. This is in line with the international literature, which implies that there are many international clinical guidelines for various conditions, providers, and settings. However, in the present study, we could include only one guideline in accordance with the ADAPTE process, the NICE CG150, for adaptation to the regional setting. According to the results of the quality evaluation and up-to-date status test results, only the NICE CG150 scored more than 50\% in the "Rigour of development" domain of K-AGREE II and included the evidence from recent 5 years at the time of development or renewal. Rigorous methodology is both the basis of high-quality guidelines and an essential factor affecting the successful adaptation of the guidelines. ${ }^{13,14}$ In addition, up-todate knowledge must be reflected in the guidelines. For example, in the past 5 years, the recommendation regarding gabapentin for treating headache has been changed because gabapentin and gabapentin enacarbil have been reported to have effects no better than placebo for prophylactic treatment of migraine in adults and are commonly associated with adverse events. ${ }^{15,16}$ As an additional example, the American Academy of Neurology guideline for episodic migraine prevention in adults, which was published in 2012, has been retired by the American Academy of Neurology Board of Directors in September, 2015, due to serious safety concerns regarding butterbur, a herb, recommended as a prophylactic treatment by this guideline. ${ }^{17}$

In summary, this paper provides detailed insight into the use of the ADAPTE process in the development of a guideline for headache. The goal of this guideline is to improve the appropriateness of diagnosis and treatment of headache disorders, and improve pain control and quality of life of patients with headache. The recommendations should be applied to clinical sites to achieve the ultimate goal of this guideline; therefore, follow-up activities, such as monitoring of guideline usage and assessment of applicability of the recommendations, should be performed in the future. Further studies on guideline reporting standards and methodological quality is necessary. It is also necessary to assess the effectiveness of this guideline in Korea.

\section{Acknowledgments}

The Korean Psychosomatic Society participated in the guideline development process.
This research was supported by a grant from the Korea Health Technology R\&D Project through the Korea Health Industry Development Institute (KHIDI), funded by the Ministry of Health \& Welfare, Republic of Korea (grant number: $\mathrm{HC17C0117).}$

\section{REFERENCES}

1. Steiner TJ, Stovner LJ, Katsarava Z, Lainez JM, Lampl C, Lanteri-Minet $\mathrm{M}$, et al. The impact of headache in Europe: principal results of the Eurolight project. J Headache Pain 2014;15:31.

2. Healthcare Bigdata Hub. Available at: http://opendata.hira.or.kr/home. do. Accessed November 1, 2018.

3. Headache Classification Committee of the International Headache Society (IHS). The international classification of headache disorders, 3rd edition (beta version). Cephalalgia 2013;33:629-808.

4. Stovner L, Hagen K, Jensen R, Katsarava Z, Lipton R, Scher A, et al. The global burden of headache: a documentation of headache prevalence and disability worldwide. Cephalalgia 2007;27:193-210.

5. Sharma TL. Common primary and secondary causes of headache in the elderly. Headache 2018;58:479-484.

6. Munksgaard SB, Jensen RH. Medication overuse headache. Headache 2014;54:1251-1257.

7. ADAPTE Collaboration. The ADAPTE process: resource toolkit for guideline adaptation. Version 2.0. 2009. Available at: https://www.g-i-n. net/. Accessed August 8, 2018.

8. Steering Committee for Clinical Practice Guideline. Korean Appraisal of Guidelines for Research \& Evaluation II. Seoul: Steering Committee for Clinical Practice Guideline; 2009.

9. Kim S, Choi M, Shin S, Ji S, Park J, Yoo J, et al. NECA's Handbook for Clinical Practice Guideline Developer. Seoul: National Evidence-based Healthcare Collaborating Agency (NECA); 2015.

10. National Institute for Health and Care Excellence (NICE). Headaches in Over 12s: Diagnosis and Management. Manchester: NICE; 2012.

11. Organization for Economic Cooperation and Development (OECD). Health at a Glance 2017: OECD Indicators. Paris: OECD Publishing; 2017.

12. Harrison MB, Legare F, Graham ID, Fervers B. Adapting clinical practice guidelines to local context and assessing barriers to their use. CMAJ 2010;182:E78-E84.

13. Brouwers MC, Kho ME, Browman GP, Burgers JS, Cluzeau F, Feder G, et al. AGREE II: advancing guideline development, reporting and evaluation in health care. CMAJ 2010;182:E839-E842.

14. Rosenfeld RM, Shiffman RN, Robertson P. Clinical Practice Guideline Development Manual, Third Edition: a quality-driven approach for translating evidence into action. Otolaryngol Head Neck Surg 2013;148:S1S55.

15. Linde M, Mulleners WM, Chronicle EP, McCrory DC. Gabapentin or pregabalin for the prophylaxis of episodic migraine in adults. Cochrane Database Syst Rev 2013:CD010609.

16. Silberstein S, Goode-Sellers S, Twomey C, Saiers J, Ascher J. Randomized, double-blind, placebo-controlled, phase II trial of gabapentin enacarbil for migraine prophylaxis. Cephalalgia 2013;33:101-111.

17. Holland S, Silberstein SD, Freitag F, Dodick DW, Argoff C, Ashman E, et al. Evidence-based guideline update: NSAIDs and other complementary treatments for episodic migraine prevention in adults: report of the Quality Standards Subcommittee of the American Academy of Neurology and the American Headache Society. Neurology 2012;78: 1346-1353. 